\title{
Result Analysis of Image Segmentation using Hierarchical Merge Tree
}

\author{
Ankit Bihone \\ M. Tech Scholar \\ Oriental College of Technology Bhopal
}

\author{
Imran Khan \\ Associate Professor \\ Oriental College of Technology Bhopal
}

\begin{abstract}
This paper aims to advance research in image segmentation by developing robust techniques for evaluating image segmentation algorithms. The key contributions of this work are as follows. First, we investigate the characteristics of existing measures for supervised evaluation of automatic image segmentation algorithms. We show which of these measures is most effective at distinguishing perceptually accurate image segmentation from inaccurate segmentation. Second, we develop a complete framework for evaluating interactive segmentation algorithms by means of user experiments. We explore four strategies for this simulation, and demonstrate that the best of these produces results very similar to those from the user experiments.
\end{abstract}

\section{Keywords}

Image Segmentation, Clustering, Region-Based, RSST Recursive Shortest-Spanning Tree.

\section{INTRODUCTION}

Image segmentation is critical for many computer vision and information retrieval systems, and has received significant attention from industry and academia over the last 30 years. Despite notable advances in the area, there is no standard technique for selecting a segmentation algorithm to use in a particular application, nor even is there an agreed upon means of comparing the performance of one method with another. This deficiency is likely a result of the inherent ambiguity in what is understood as the purpose and scope of segmentation itself.[2]

Like many complex computer vision problems, image segmentation is ill-defined. A common, if rather unconstrained, definition of segmentation is that it is the process of partitioning the set of pixels in an image into several disjoint subsets, according to a set of predefined criteria. Although this definition admits and conforms to almost all other definitions found in the literature, the criteria itself is usually a source of debate.

It defines image segmentation as the process of dividing an image into different regions such that each region is, but the union of any two adjacent regions is not, homogeneous. Similarly, Morris et. al.[9] describes segmentation as the process of partitioning an image into regions that are in some sense homogeneous, but different from neighboring regions. Skarbek and Koschan[13] for a simpler interpretation: the identification of homogeneous regions. All these definitions use the concept of homogeneity, which usually corresponds to identifying regions containing features that are relatively nearby according to a prescribed distance measure.

Segmentation may also be considered as an algorithmic attempt to mimic a human interpretation of an image, known as perceptual grouping. Considering segmentation in this way substantially increases the scope and complexity of the problem. Fu and Mui [21] and assume this viewpoint, stating that "the image segmentation problem is basically one of psychophysical perception, and therefore not susceptible to a purely analytical solution." It also implies this interpretation in their work on comparing automatic segmentation algorithms with human generated ground truth. It both argues that perceptual grouping is hierarchical in nature, and consequentially a flat partitioning of an image is insufficient for representing a perceptual segmentation.

It is clear from the above that there is considerable variation in what is understood to be the scope and definition of the image segmentation problem. Image segmentation is usually one of several components in a larger information processing system, and the variation observed in the definition of image segmentation is mirrored in the variation in requirements on the image segmentation algorithms in these systems. For multimedia information retrieval systems, [5] image segmentation algorithms capable of producing homogeneous regions usually suffice, since the purpose of image segmentation in such systems is often simply to create a set of localized features. Object recognition systems, on the other hand, usually require semantic objects from which features can be extracted and processed by pattern recognition engine (a support vector machine, for example).[7] In some cases, a priori information about the object is available, or can be fed back into the segmentation algorithm; in other cases, no such information is available, and the segmentation algorithm is required to produce regions or objects based on the image data alone.

\subsection{Supervised Image Segmentation}

The Supervised segmentation is an approach where the model parameters are assumed to be known a priori and are used for estimating the pixel labels in segmentation problem. The pixel labeling problem, using MRF model has been formulated using maximum a posteriori (MAP) criterion and Bayesian framework [11-9]. Segmentation of both noisy and textured images could be formulated in supervised frame work using MRF model. For Brain MR images, D. Patra[15] have proposed Hybrid Tabu Search (HTS) algorithm to obtain the MAP estimates of the image labels and thus to accomplish supervised image segmentation.

\subsection{Unsupervised Image Segmentation}

In unsupervised framework, the number of class labels and model parameters are unknown and are to be estimated simultaneously. The unsupervised image segmentation is viewed as the incomplete data problem as the estimation of image labels depend upon the optimal set of parameters and vice versa. This type of problem is usually addressed using iterative schemes such as iterative conditional mode (ICM) algorithm which was initiated by Besag [22]. 


\section{METHOD}

\subsection{Fast Fuzzy C-means clustering}

This research aims at decreasing the number of distance calculations of the FCM by computing the distances between data points and the nearest cluster centres for points with membership values greater than a threshold, $T$, where the value of $\mathrm{T}$ is less than 1 and greater than 0 .

In this case, there is no need to calculate distances for points with membership values less than $\mathrm{T}$ since these values do not severely affect the results and therefore, some distance calculations can be saved. To illustrate the FFCM algorithm.

The Fuzzy C-means Clustering (FCM) algorithm is a data clustering algorithm in which each data point belongs to a cluster to a degree specified by a membership grade.

FCM partitions a collection of $n$ data points $x_{i}, i=1, \ldots, n$ into c fuzzy groups, and finds a cluster center in each group such that a cost function of dissimilarity measure is minimized. The major Proceedings of the 6th WSEAS Int. Conf. on Artificial Intelligence, Knowledge Engineering and Data Bases, Corfu Island, Greece, February 16-19, 200728 difference between FCM and hard clustering is that FCM employs fuzzy partitioning such that a given data point can belong to several groups with the degree of belongingness specified by the membership grades between 0 and 1 . The membership matrix $\mathrm{U}$ is allowed to have elements with values between 0 and 1 . However, imposing normalization stipulates that the summation of degrees of belongingness for a data set always be equal to unity:

$$
\sum_{i=1}^{c} u_{i j}=1, \forall j=1
$$

The cost function (or objective function) for FCM is:

$$
J\left(U, c_{1}, \ldots \ldots \ldots, c_{c}\right)=\sum_{i=1}^{c} j_{i}=\sum_{j}^{n} u_{i j}^{m} d_{i j}^{2}
$$

where $u_{i j}$ uij is between 0 and $1 ; c_{i}$ is the cluster center of fuzzy group $\mathrm{i} ; d_{i j}=\left\|c_{i}-x_{j}\right\|$ is the Euclidean distance between ith cluster and jth data point; and $m \in[1, \infty)$ is weighting exponent.

The necessary conditions for Equation (2) to reach its minimum are:

$$
\begin{gathered}
c_{i}=\frac{\sum_{j=1}^{n} u_{i j}^{m} x_{j}}{\sum_{j=1}^{n} u_{i j}^{m}} \\
u_{i j}=\frac{1}{\sum_{k=1}^{c}\left(\frac{d_{i j}}{d_{k j}}\right)^{2 /(m-1)}}
\end{gathered}
$$

The fuzzy C-means algorithm is simply an iterated procedure through the preceding two necessary conditions.

\section{RESULT}

In this research work we proposed algorithm and proposed techniques are applied on the input of proposed work then we get output in graphical image are as follows:

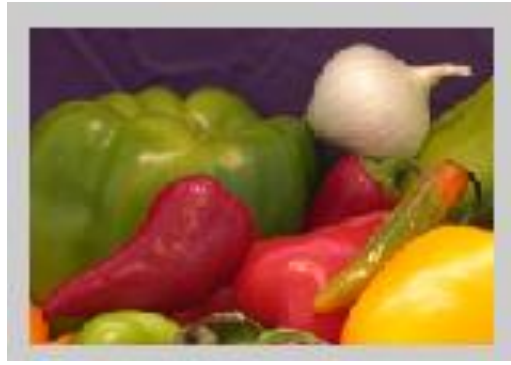

Fig.1 original input image

This fig. 1 shows the original input image, which take the form of input of our proposed work.

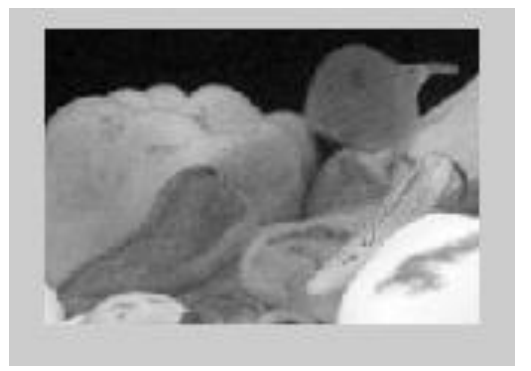

Fig.2 image segmentation

This fig. 2 shows the image segmentation. In this process being segmentation of input image.

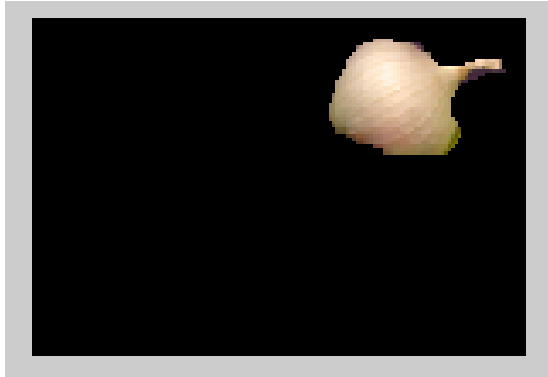

Fig.3 Fast Fuzzy C-means clustering image

This fig. 3 shows the clustering image. This image we obtain after applied fast fuzzy $\mathrm{C}$-means clustering image.

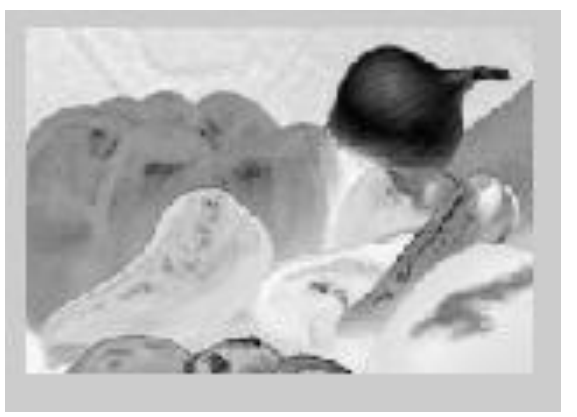

Fig.4 color segmentation

This fig.4 shows the color segmented image. This image we get after color segmentation. 


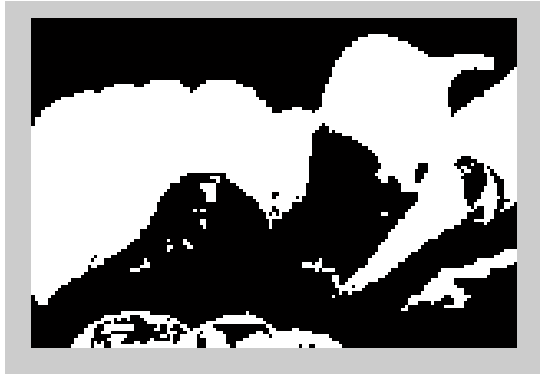

Fig.5 Output image

This fig. 5 shows the output image. This image we obtained after applied the proposed algorithm on input image for the better result.

\section{CONCLUSION}

This conclusion summarizes the research, outlines the key contributions, suggests potential directions for future research, and notes our related publications to date. The experimental results show that each algorithm has advantageous properties as well as some specific drawbacks. None of them gives a complete solution to this challenging problem. We also introduce a modification to the hierarchical merge tree model that iteratively trains a new boundary classifier with accumulated samples for merge tree construction and merging probability prediction and accumulates segmentation to generate contour maps.

\section{REFERENCES}

[1] Ting Liu, Mojtaba Seyedhosseini, and Tolga Tasdizen "Image Segmentation Using Hierarchical Merge Tree" IEEE transactions on image processing, vol. 25, no. 10, october 2016

[2] John M. Gauch "Image Segmentation and Analysis via Multiscale Gradient Watershed Hierarchies" IEEE transactions on image processing, vol. 8, no. 1, January 1999

[3] Kostas Haris, Serafim N. Efstratiadis, Nicos Maglaveras Aggelos K. Katsaggelos "Hybrid Image Segmentation Using Watersheds and Fast Region Merging" IEEE transactions on image processing, vol. 7, no. 12, december 1998

[4] Jianbo Shi, Jitendra Malik "Normalized Cuts and Image Segmentation" IEEE Transactions on Pattern Analysis and Machine Intelligence, Volume 22, Issue 8, August 2000 , pages 888-905.

[5] Chiou-Shann Fuh, Shun-Wen Cho, and Kai Essig "Hierarchical Color Image Region Segmentation for Content-Based Image Retrieval System" IEEE transactions on image processing, vol. 9, no. 1, January 2000

[6] M. Seyedhosseini and T. Tasdizen, "Semantic image segmentation with contextual hierarchical models," IEEE Trans. Pattern Anal. Mach. Intell.,vol. 38, no. 5, pp. 951964, May 2015.

[7] S. Belongie, C. Carson, H. Greenspan, and J. Malik, "Color- and texturebased image segmentation using EM and its application to contentbased image retrieval," in Proc. Int. Conf. Comput. Vis., Jan. 1998, pp. 675-682.
[8] D. Comaniciu and P. Meer, "Mean shift: A robust approach toward feature space analysis," IEEE Trans. Pattern Anal. Mach. Intell., vol. 24, no. 5, pp. 603-619, May 2002.

[9] O.J. Morris, M.de J.Lee, and A.G. Constantinides, 1986. A unified method for segmentation and edge detection using graph theory. Proc. ICASSP, pp. 2051-2054.

[10] A. Vedaldi and S. Soatto, "Quick shift and kernel methods for mode seeking," in Proc. Eur. Conf. Comput. Vis., 2008, pp. 705-718.

[11] J. Shi and J. Malik, "Normalized cuts and image segmentation," IEEE Trans. Pattern Anal. Mach. Intell., vol. 22 , no. 8, pp. 888-905, Aug. 2000.

[12] P. F. Felzenszwalb and D. P. Huttenlocher, "Efficient graph-based image segmentation,” Int. J. Comput. Vis., vol. 59, no. 2, pp. 167-181, Sep. 2004.

[13] W. Skarbek, A. Koschan, Colour Image Segmentation A Survey, Technical Report 94-32, Technical University Berlin, October 1994.

[14] H. Zhu, F. Meng, J. Cai, and S. Lu, "Beyond pixels: A comprehensive survey from bottom-up to semantic image segmentation and cosegmentation," J. Vis. Commun. Image Represent., vol. 34, pp. 12-27, Jan. 2016.

[15] Dipti Patra. Brain MR image segmentation using Markov random field model and Tabu search strategy. $\mathrm{PhD}$ thesis, National Institute of Technology, Rourkela, India, Department of Electrical Engineering, 2005.

[16] D. Martin, C. Fowlkes, D. Tal, and J. Malik, “A database of human segmented natural images and its application to evaluating segmentation algorithms and measuring ecological statistics," in Proc. 8th IEEE Int. Conf. Comput. Vis. (ICCV), vol. 2. Jul. 2001, pp. 416-423.

[17] J. Shotton, J. Winn, C. Rother, and A. Criminisi, "TextonBoost: Joint appearance, shape and context modeling for multi-class object recognition and segmentation," in Proc. Eur. Conf. Comput. Vis., 2006, pp. 1-15.

[18] S. Gould, R. Fulton, and D. Koller, "Decomposing a scene into geometric and semantically consistent regions," in Proc. Int. Conf. Comput. Vis., Sep./Oct. 2009, pp. 1-8.

[19] M. Everingham, L. Van Gool, C. K. I. Williams, J. Winn, and A. ZissermanThe PASCAL Visual Object Classes Challenge 2012 (VOC2012) Results. acessed on Dec. 9, $2014 . \quad$ [Online]. Available:http://www.pascalnetwork.org/challenges/VO C/voc2012/workshop/index.html

[20] N. Silberman, D. Hoiem, P. Kohli, and R. Fergus, "Indoor segmentation and support inference from RGBD images," in Proc. Eur. Conf. Comput. Vis., 2012, pp. 746-760.

[21] K. S. Fu and J. K. Mui, A survey on image segmentation, Pattern Recognir. 13, 1981, 3-16.

[22] J. Besag. On the statistical analysis of dirty pictures. Journal of Royal Statistical Society B, 62:259-302, 1986. 